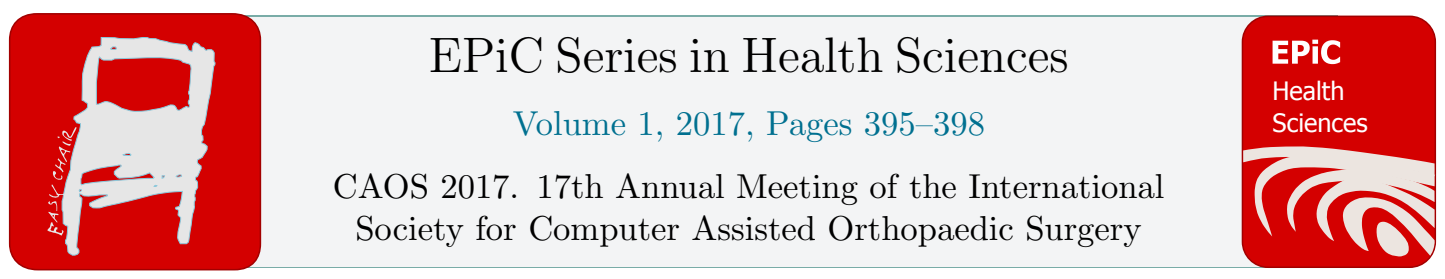

\title{
Change in pelvic sagittal tilt after corrective long fusion of spine
}

\author{
Tamura K, Takao M, Sakai T, Hamada H, Kaito T, Sugano N \\ 1Department of Orthopaedic Medical Engineering, Osaka University Graduate School of Medicine, Suita, Osaka, Japan \\ 2 Department of Orthopaedic Surgery, Osaka University Graduate School of Medicine, Suita, Osaka, Japan \\ coo_kaz_cootan@yahoo.co.jp
}

\begin{abstract}
The individual pelvic sagittal inclination (PSI) is an issue for cup alignment in total hip arthroplasty (THA). The chronological PSI change is larger in standing than that in supine. It is expected that PSI in standing position may improve when spinal long fusion was performed. Therefore, the purpose of this study was to evaluate the change of PSI between pre- and post- corrective long fusion for adult spinal deformity (ASD). Total eight patients underwent corrective surgery for ASD between May 2014 and October 2016 were the subjects of this study. There were two cases that underwent bilateral THA before spine surgery. PSI was defined as the angle between the anterior pelvic plane and the vertical axis on the sagittal plane DRR. The mean change in the preoperative PSI from supine to standing was $17^{\circ}$ posteriorly. The mean change in the supine PSI from pre- to post-operation was $6.9^{\circ}$ anteriorly, and that in the standing PSI was $17^{\circ}$ anteriorly. The mean change in the postoperative PSI from supine to standing was $6.7^{\circ}$ posteriorly. When we measured PSI on standard AP radiographs of the pelvis in two patients who underwent THA, the mean change in postoperative PSI from supine to standing was less than $3^{\circ}$.The mean change in cup anteversion on supine AP radiographs were $2.7^{\circ}$. As we expected, the change in PSI from supine to standing position was reduced by performing corrective surgery. In this study, PSI in standing changed into the direction which reduce posterior impingement and anterior dislocation..
\end{abstract}

\section{Introduction}

The individual pelvic sagittal inclination (PSI) is an issue for cup alignment in total hip arthroplasty (THA). The chronological PSI change is larger in standing than that in supine. It is expected that PSI in standing position may improve when spinal long fusion was performed. Therefore, the purpose of this study was to evaluate the change of PSI between pre- and postcorrective long fusion for adult spinal deformity (ASD). Total eight patients underwent corrective surgery for ASD between May 2014 and October 2016 were the subjects of this study. There were 
two cases that underwent bilateral THA before spine surgery. PSI was defined as the angle between the anterior pelvic plane and the vertical axis on the sagittal plane DRR. The mean change in the preoperative PSI from supine to standing was $17^{\circ}$ posteriorly. The mean change in the supine PSI from pre- to post-operation was $6.9^{\circ}$ anteriorly, and that in the standing PSI was $17^{\circ}$ anteriorly. The mean change in the postoperative PSI from supine to standing was $6.7^{\circ}$ posteriorly. When we measured PSI on standard AP radiographs of the pelvis in two patients who underwent THA, the mean change in postoperative PSI from supine to standing was less than $3^{\circ}$.The mean change in cup anteversion on supine AP radiographs were $2.7^{\circ}$. As we expected, the change in PSI from supine to standing position was reduced by performing corrective surgery. In this study, PSI in standing changed into the direction which reduce posterior impingement and anterior dislocation.

\section{Materials and Methods}

Total eight patients underwent corrective surgery for ASD between May 2014 and October 2016 were the subjects of this study. All are females with the average age of 70 years (range, 59 to 77). There were two cases that underwent bilateral THA before spine surgery. Standardized anteroposterior (AP) and lateral digital x-rays of the full-length spine in standing and supine were obtained at pre- and post-operation. AP and lateral digitally reconstructed radiographs (DRR) of the pelvis were created from individual CT images using 3D viewer software (3D Template; Kyocera Medical, Osaka, Japan). The anterior pelvic plane (APP) through the most anterior aspect of the pubic tubercle and bilateral anterior superior iliac spines (ASISs) was used to measure PSI, which was defined as the angle between the APP and the vertical axis on the sagittal plane DRR. PSI on the standing and supine radiographs were calculated using a 2D-3D matching technique with CT images and pre- and post-operative AP radiographs, as reported previously. (Nishihara 2003) The pelvis was rotated on the sagittal plane on the 3D viewer to match the shape of the pelvis on the AP DRR with that on the AP radiograph. (Fig.1) PSI on the AP radiograph was then measured on the sagittal plane DRR. (Fig.2) In addition, we measured the cup anteversion in two THA cases by using viewer software (2D Template; Kyocera Medical, Osaka, Japan).

\section{Results}

The mean change in the preoperative PSI from supine to standing was $17^{\circ}$ posteriorly. The mean change in the supine PSI from pre- to post-operation was $6.9^{\circ}$ anteriorly, and that in the standing PSI was $17^{\circ}$ anteriorly. The mean change in the postoperative PSI from supine to standing was $6.7^{\circ}$ posteriorly. When we measured PSI on standard AP radiographs of the pelvis in two patients who underwent THA, the mean change in postoperative PSI from supine to standing was less than $3^{\circ}$, and standing PSI became closer to that in supine. The mean change in cup anteversion on supine AP radiographs were $3.4^{\circ}\left(2.7^{\circ}\right.$ to $\left.3.5^{\circ}\right)$.

\section{Discussion}

As we expected, the change in PSI from supine to standing position was reduced after spinal corrective surgery by $13^{\circ}$. When we measured with standard AP radiographs of the pelvis who underwent THA, change in the supine PSI between pre- and post-operation was less than $5^{\circ}$. In this study, the mean change of radiographic cup anteversion in supine after spinal corrective surgery was $3.2^{\circ}$ and PSI in standing changed into the direction which reduce posterior impingement and anterior 
dislocation. Although the change in PSI after spinal corrective surgery heavily depends on the spine surgeon's philosophy of correction (PI-LL $<20^{\circ}, \mathrm{PT}<20^{\circ}$ ). In this study the mean PI-LL was $6.3^{\circ}$ and PT was $20.5^{\circ}$ post corrective surgery. Spinal corrective surgery should have effect on cup alignment in standing and little influence on cup alignment in supine. If the spinal corrective surgery was performed in optimal alignment, therefore, when we performed THA using FPP as reference plane for positioning of the acetabular component before spinal corrective surgery, there is little risk of implant impingement.

\section{References}

Sugano N, Nishii T, Miki H, Yoshikawa H, Sato Y, Tamura S. Mid-term results of cementless total hip replacement using a ceramic-on-ceramic bearing with and without computer navigation. $\mathrm{J}$ Bone Joint Surg Br. 2007 Apr;89(4):455-60.

Miki H, Kyo T, Sugano N, Anatomical Hip Range of Motion After Implantation During Total Hip Arthroplasty With a Large Change in Pelvic Inclination. J Arthroplasty. 2012; Vol.27 No.9 October

Barrey C, Jund J, Noseda O, Roussouly P, Sagittal balance of the pelvis-spine complex and lumbar degenerative diseases. A comparative study about 85 cases. Eur Spine J 2007:16:1459-1467.

Tamura S, Miki H, Tsuda K, Takao M, Hattori A, Suzuki N, Yonenobu K, Sugano N. Hip range of motion during daily activities in patients with posterior pelvic tilt from supine to standing position. J Orthop Res. 2015 Apr;33(4):542-7.

Tamura S, Nishihara S, Takao M, Sakai T, Miki H, Sugano N. Does Pelvic Sagittal Inclination in the Supine and Standing Positions Change Over 10 Years of Follow-Up After Total Hip Arthroplasty? J Arthroplasty 2016, September 9

Nishihara S, Sugano N, Nishii T, Ohzono K, Yoshikawa H. Measurements of pelvic flexion angle using three-dimensional computed tomography. Clin Orthop Relat Res. 2003; (411):140-151. 


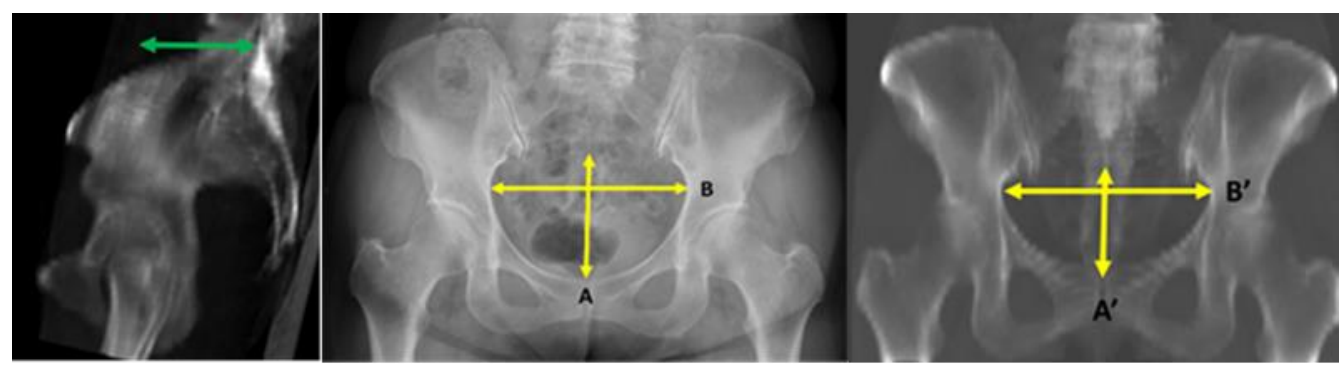
(a). sagittal DRR
(b). full-length radiograph
(c). AP DRR

Figure 1. 2D-3D matching technique with $C T$ images. Rotated sagittal DRR view (a) until A:B (b) = A': B' (c)

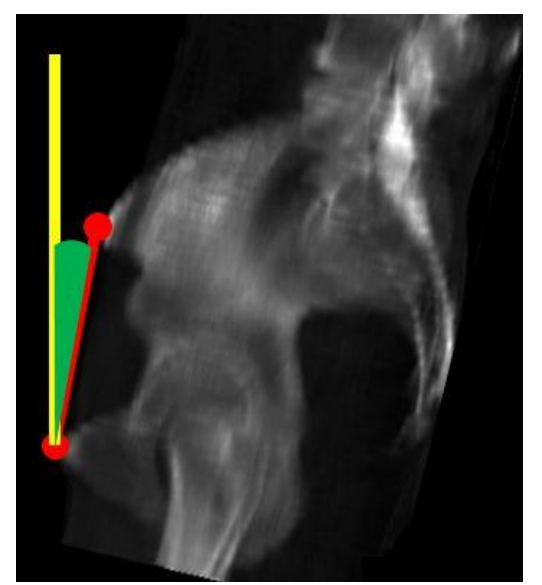

Figure 2. PSI (green angle) was defined as the angle between the APP (red line) and the vertical axis (yellow line) 